\title{
Infinite Sums of Roots for a Class of Transcendental Equations and Bessel Functions of Order One-Half
}

\author{
By N. Liron
}

\begin{abstract}
The roots of Bessel functions of order one-half are special cases of roots of transcendental equations of the form $\tan z=A(z) / B(z)$, where $A(z), B(z)$ are polynomials and $A(z) / B(z)$ is odd. We prove that the function $f(z)=B(z) \sin z-A(z) \cos z, f(z)$ even or odd, satisfies the conditions of Hadamard's factorization theorem, and derive recurrences for sums of the form $S_{l}=\sum_{k=1}^{\infty} \alpha_{k}^{-2 l}, l=1,2, \cdots$, where the $\alpha_{k}$ 's are the nonzero roots of $f(z)$. We also prove under what conditions on $A(z)$ and $B(z)$ is $S_{l}=\pi^{-2 l-2} \xi(2 l+2)$ or $S_{l}=\pi^{-2 l-2 \zeta}(2 l+2)\left(2^{2 l+2}-1\right)$, where $\zeta$ is the Riemann zeta function. We prove that, although Bessel functions of positive half-order, $J_{l+1 / 2}$, have only real roots, perturbation of any one of its coefficients introduces nonreal roots for $l>2$.
\end{abstract}

1. Introduction. We are interested in sums of the form

$$
S_{k}=\sum_{n=1}^{\infty} \alpha_{n}^{-2 k-2}
$$

where the $\alpha_{n}$ 's are the nonzero roots of a function of the type

$$
f(z)=B_{m}(z) \sin z-A_{n}(z) \cos z,
$$

where $B_{m}(z), A_{n}(z)$ are polynomials of order $m, n$, respectively, $m \neq n$, and $f(z)$ is either even or odd. Since the roots occur in pairs, $\pm \alpha$, we take only one of each pair.

Two special cases of (1.2), $B_{m}(z)=1, A_{n}(z)=k z$, and $B_{m}(z)=z, A_{n}(z)=-k$, $k$ a nonzero real constant, have been treated in [1] using Sturm-Liouville theory.

We shall show that (1.2) has a discrete sequence of roots, $\alpha_{n}$, with $\alpha_{n}^{2} \rightarrow \infty$. As a special case, we get the Bessel functions $J_{l+1 / 2}(z)$ and $J_{-l-1 / 2}(z)$, for $l>0$. For $J_{l+1 / 2}(z)$ we prove, by using $S_{0}$, that if $l>2$, although $J_{l+1 / 2}(z)$ itself has only real roots, perturbations of any one of its coefficients (written in the form (1.2)), introduce nonreal roots.

\section{Main Theorem.}

LEMMA 1. Let

$$
f(z)=B_{m}(z) \sin z-A_{n}(z) \cos z, \quad m \neq n,
$$

where $B_{m}(z), A_{n}(z)$ are polynomials of order $m, n$, respectively, which have no common root.

Received November 30, 1970.

AMS 1970 subject classifications. Primary 30-00, 30A08, 33A40, 65H05; Secondary $10 \mathrm{H} 05$.

Key words and phrases. Bessel functions of order one-half, roots of a transcendental equation, Hadamard's factorization theorem, infinite sums of powers of roots, recurrence relations, multiplicity and splitting of roots at and from zero, Sturm-Liouville system.

Copyright @ 1971, American Mathematical Society 
Let $z=x+i y$. Then

(1) There exists a $Y>0$, such that if $y>Y, f(z)$ has no roots.

(2) In any strip $|x| \leqq L<\infty$, there can only be a finite number of roots of $f(z)$.

Proof. Part (1). $f(z)=0$ iff

$$
\tan z=A_{n}(z) / B_{m}(z),
$$

(including both sides $=\infty$ ). For $|z| \gg 1$,

$$
\left|A_{n}(z) / B_{m}(z)\right| \sim C|z|^{n-m}=C|z|^{k},
$$

where $k=n-m$ and $C>0$. If $|z| \gg 1$, we must therefore have

$$
|\tan z| \approx C|z|^{k}
$$

if $z$ is a root. But

$$
\begin{aligned}
\tan z & =\tan (x+i y)=\frac{\tan x+i \tanh y}{1-i \tan x \tanh y} \\
& =\frac{\sin x \cos x\left(1-\tanh ^{2} y\right)+i \tanh y}{\cos ^{2} x+\sin ^{2} x \tanh ^{2} y},
\end{aligned}
$$

from which it follows that

$$
|\tanh y| \leqq|\tan z| \leqq \frac{1}{2}+\frac{1}{2 \tanh ^{2} y},
$$

i.e., $\tan z$ is bounded away from zero and infinity, if $y \neq 0$. If $|y| \gg 1$, then (2.5) implies that $|\tan z| \approx 1$. But if $|y| \gg 1$, then $|z| \gg 1$, and (2.4) holds for a root, i.e., $|\tan z| \gg 1$ for $k>0$, and $|\tan z| \ll 1$ for $k<0$.

This concludes the proof of (1).

Part (2). Suppose we had an infinite number of roots for $|x| \leqq L<\infty$. By Part (1), they would be in a bounded domain, and would have an accumulation point other than infinity. Since $f(z)$ is an analytic entire function, it follows that $f(z) \equiv 0$, by Taylor's Theorem. This contradiction proves Part (2).

Di Remark. It follows from (2.4) that we do have an infinite sequence of roots, tending to $\pm \infty$ on the real line, with the asymptotic values $n \pi$ for $k<0$ and $\left(n+\frac{1}{2}\right) \pi$ for $k>0, n$ an integer.

LEMMA 2. Let $\lambda \equiv$ order of $f(z)$. Then $\lambda \leqq 1$.

Proof. Let $M(r)=\operatorname{Max}_{|z|=r}|f(z)|$. Then, $M(r) \leqq(n+m) C_{1} r^{m+n} e^{r}$, for $r>1$, and $C_{1}$ is the largest coefficient in absolute value of $A_{n}(z)$ and $B_{m}(z)$.

$$
\begin{aligned}
\log M(r) & \leqq \log \left[(n+m) C_{1}\right]+(m+n) \log r+r \\
& \leqq C_{2} r, \quad \text { for } r \text { large enough and } C_{2}>0 .
\end{aligned}
$$

So that, $\log \log M(r) \leqq \log C_{2}+\log r$, and therefore,

$$
\lambda=\limsup _{r \rightarrow \infty} \frac{\log \log M(r)}{\log r} \leqq 1+\lim _{r \rightarrow \infty} \frac{\log C_{2}}{\log r}=1 .
$$

TheOREM 1. Let $f(z)=B_{m}(z) \sin z-A_{n}(z) \cos z$, where $B_{m}(z), A_{n}(z)$, are polynomials of order $m, n$, respectively, one being even and the other odd. Then 


$$
f(z)=C_{0} z^{q} \prod_{k=1}^{\infty}\left(1-z^{2} / \alpha_{k}^{2}\right),
$$

where $\alpha_{k}$ are the roots of $f(z)=0$, such that $\left|\alpha_{1}\right| \leqq\left|\alpha_{2}\right| \leqq \cdots \leqq\left|\alpha_{n}\right| \leqq \cdots, q$ is the multiplicity of the root $z=0$, and $C_{0}=f^{(a)}(0) / q$ !

Proof. From Lemma 1 and the following remark we get that there exists an infinite sequence of roots of $f(z)=0$, accumulating only at infinity. Also, the roots occur in pairs $\pm \alpha$. By Hadamard's factorization theorem [2, p. 22], we can write

$$
f(z)=C_{0} \exp \{g(z)\} z^{\alpha} \prod_{k=1}^{\infty}\left(1-z^{2} / \alpha_{k}^{2}\right), \quad C_{0} \neq 0,
$$

where $q ! C_{0}=f^{(q)}(0)$, and $g(z)$ is a polynomial in $z$ of order $\leqq \lambda$, with $g(0)=0$. By Lemma $2, \lambda \leqq 1$, and we get

$$
g(z)=a z .
$$

Since $f(z)$ is even or odd, we get that $q$ must be even or odd with $f(z)$, and $\exp \{g(z)\}=$ $\exp \{g(-z)\}$, i.e., $a z=-a z$ by (2.8), or $a=0$, which proves (2.6).

3. Recurrences and Special Cases of $S_{k}$. Rewrite $f(z)$ in Theorem 1 as

$$
f(z)=b_{l+2 m}(z) \sin z-a_{p+2 n}(z) \cos z,
$$

where

$$
b_{l+2 m}(z)=z^{l} \sum_{k=0}^{m} b_{k} z^{2 k}=z^{l} B_{m}\left(z^{2}\right), \quad b_{0} \neq 0, b_{m} \neq 0,
$$

and

$$
a_{p+2 n}(z)=z^{\mathcal{D}} \sum_{k=0}^{n} a_{k} z^{2 k}=z^{\mathcal{D}} A_{n}\left(z^{2}\right), \quad a_{0} \neq 0, a_{n} \neq 0,
$$

and $l+p$ is an odd integer.

Since we are interested in the nonzero roots of $f(z)$, we can divide ${ }^{\prime} y_{-}^{\prime} b_{0} z^{\operatorname{Min}(l, p)}$. The two basic cases are therefore:

$$
f(z)=B_{m}\left(z^{2}\right) \sin z-z^{+} A_{n}\left(z^{2}\right) \cos z, \quad r=2 t+1>0,
$$

and

$$
f(z)=z^{r} B_{m}\left(z^{2}\right) \sin z-A_{n}\left(z^{2}\right) \cos z, \quad r=2 t+1>0,
$$

with $b_{0}=1$ in both cases.

Case 1. Consider $f(z)$ as in (3.4). Write

$$
\begin{aligned}
& B_{m}\left(z^{2}\right) \sin z=\sum_{s=0}^{\infty} c_{s} z^{2 s+1}, \\
& A_{n}\left(z^{2}\right) \cos z=\sum_{s=0}^{\infty} d_{s} z^{s},
\end{aligned}
$$

from which we get 


$$
\begin{aligned}
& c_{s}=(-1)^{s} \sum_{k=0}^{m}(-1)^{k} b_{k} /(2 s-2 k+1) !, \\
& d_{s}=(-1)^{s} \sum_{k=0}^{n}(-1)^{k} a_{k} /(2 s-2 k) !, \quad s=0,1,2, \cdots
\end{aligned}
$$

Substitute (3.6), (3.7) into (3.4) to get

$$
f(z)=\sum_{s=0}^{\infty} c_{s} z^{2 s+1}-\sum_{s=0}^{\infty} d_{s} z^{2 s+r},
$$

and by (2.6) we can write

$$
\begin{aligned}
& f(z)=C_{0} z^{a} \prod_{k=1}^{\infty}\left(1-z^{2} / \alpha_{k}^{2}\right), \\
& \text { where } C_{0}=c_{0}, q=1 \text { for } r>1, \\
& \qquad \begin{aligned}
C_{0}=c_{i}-d_{i}, q=2 i+1 \text { for } r=1, c_{i}-d_{i}=0, \\
j=0,1, \cdots, i-1, \text { and } c_{i}-d_{i} \neq 0 .
\end{aligned}
\end{aligned}
$$

Take the logarithmic derivatives of (3.9), (3.10), equate, and multiply by $z f(z), f(z)$ as in (3.9) to get

$$
\begin{aligned}
\sum_{s=0}^{\infty}(2 s+1) c_{s} z^{2 s+1} & -\sum_{s=0}^{\infty} d_{s}(2 s+r) z^{2 s+r} \\
& =\left[q-2 \sum_{s=0}^{\infty} S_{s} z^{2 s+2}\right]\left[\sum_{s=0}^{\infty} c_{s} z^{2 s+1}-\sum_{s=0}^{\infty} d_{s} z^{2 s+r}\right],
\end{aligned}
$$

or, after rearranging, and dividing by 2 ,

$$
\begin{aligned}
\sum_{s=0}^{\infty} z^{2 s+3} \sum_{k=0}^{\infty} S_{k}\left[c_{s-k}-d_{s-t-k}\right]= & -\sum_{s=0}^{\infty}[s+1-(q-1) / 2] c_{s+1} z^{2 s+3} \\
& +\sum_{s=0}^{\infty}[s+1-(q-r) / 2] d_{s+1} z^{2 s+2+r},
\end{aligned}
$$

with $d_{i}=0$ for $j<0$. The coefficient of $z$ on the right-hand side is

$$
(q-1)\left[c_{0}-d_{0} \delta_{r, 1}\right] / 2=0 \text { by (3.10). }
$$

Equate coefficients of $z^{2 l+3}$ in (3.12) to get

$$
\begin{aligned}
\sum_{k=0}^{l} S_{k}\left[c_{l-k}-d_{l-t-k}\right]=-[l+1-(q-1) / 2]\left[c_{l+1}-d_{l+1-t}\right], & \\
l & =0,1,2, \cdots .
\end{aligned}
$$

Case 1.1: $t>0$. In this case $q=1$ and (3.13) becomes

$$
\sum_{k=0}^{l} S_{k}\left[c_{l-k}-d_{l-t-k}\right]=-(l+1)\left[c_{l+1}-d_{l+1-l}\right], \quad l=0,1,2, \cdots,
$$

from which we obtain, as special cases

$$
\begin{aligned}
& S_{0}=\frac{1}{6}-b_{1}+a_{0} \delta_{t, 1}, \\
& S_{1}=b_{1}^{2}-2 b_{2}+\frac{1}{90}+\left[2 a_{1}-a_{0}\left(\frac{2}{3}+2 b_{1}-a_{0}\right)\right] \delta_{t, 1}+2 a_{0} \delta_{t, 2} .
\end{aligned}
$$


TheOREM 2. Let $b_{k}=0, k=1,2, \cdots, p-1$, and $b_{p} \neq 0$. Define

$$
S_{l}=\pi^{-2 l-2} \zeta(2 l+2)+F_{l}, \quad l=0,1,2, \cdots,
$$

where $\zeta(s)=\sum_{n=1}^{\infty} n^{-s}$ is the Riemann zeta function. Then

$$
F_{l}=0, \quad l \leqq \operatorname{Min}(t-2, p-2),
$$

$$
F_{l}=(l+1)\left[a_{0} \delta_{l, t-1}-b_{p} \delta_{l, p-1}\right], \quad l=\operatorname{Min}(t-1, p-1) .
$$

Proof. If $l+1<t$ then (3.14) becomes

$$
\sum_{k=0}^{l} S_{k} c_{l-k}=-(l+1) c_{l+1} .
$$

If also $l+1<p$, we get from (3.8) that for $s \leqq l+1$,

$$
c_{s}=(-1)^{s} \frac{b_{0}}{(2 s+1) !}=\frac{(-1)^{s}}{(2 s+1) !} \text {. }
$$

So that if $l \leqq \operatorname{Min}(t-2, p-2)$, then (3.14) takes the form

$$
\sum_{k=0}^{l} \frac{(-1)^{k} S_{k}}{(2 l-2 k+1) !}=\frac{l+1}{(2 l+3) !},
$$

which is equation [1, (21)], for the parameter $k=0$. But in this case [1, (24)], we have $S_{l}=\pi^{-2 l-2} \zeta(2 l+2)$, i.e., (3.17). If $l=\operatorname{Min}(t-1, p-1)$, then (3.14) is

$$
\sum_{k=0}^{l} S_{k} c_{l-k}=-(l+1)\left[c_{l+1}-d_{0} \delta_{l, t-1}\right]
$$

or

$$
\sum_{k=0}^{l} \frac{(-1)^{l-k} S_{k}}{(2 l-2 k+1) !}=(l+1)\left[\frac{(-1)^{l}}{(2 l+3) !}-b_{p} \delta_{l, p-1}+a_{0} \delta_{l, t-1}\right] .
$$

(3.18) now follows from (3.16), (3.17) and (3.20).

Case 1.2: $t=0$. In this case, $i=(q-1) / 2$ (see (3.10)), and (3.13) is

$$
\sum_{k=0}^{l} S_{k}\left[c_{l-k}-d_{l-k}\right]=-[l+1-i]\left[c_{l+1}-d_{l+1}\right] \text {. }
$$

From (3.10), $c_{i}-d_{i}=0$ for $j<i$, and (3.21) is an identity, $0=0$, if $l<i$. For $l \geqq i$, we have

$$
\sum_{k=0}^{l-i} S_{k}\left(c_{l-k}-d_{l-k}\right)=-(l+1-i)\left[c_{l+1}-d_{l+1}\right],
$$

or, replace $l-i$ by $l$, to get

$$
\sum_{k=0}^{l} S_{k}\left[c_{l+i-k}-d_{l+i-k}\right]=-(l+1)\left(c_{l+i+1}-d_{l+i+1}\right),
$$

$$
l=0,1,2, \cdots \text {. }
$$

As special cases, we have

$$
S_{0}=-\frac{c_{i+1}-d_{i+1}}{c_{i}-d_{i}}, \quad S_{1}=\left[\frac{c_{i+1}-d_{i+1}}{c_{i}-d_{i}}\right]^{2}-2 \frac{c_{i+2}-a_{i+2}}{c_{i}-d_{i}} .
$$


A theorem similar to Theorem 2, for this case will be given in Section 4.

Case 2. Consider now $f(z)$ as in (3.5). From (3.6), (3.7), (3.8), we get

$$
f(z)=\sum_{s=0}^{\infty} c_{s} z^{2 s+1+r}-\sum_{s=0}^{\infty} d_{s} z^{2 s+1}
$$

and by (2.6), we write

$$
f(z)=C_{0} z^{a} \prod_{k=1}^{\infty}\left(1-z^{2} / \alpha_{k}^{2}\right)
$$

with $C_{0}=-a_{0}, q=0$. Repeat the same process as before to get

$$
\begin{aligned}
\sum_{s=0}^{\infty} z^{2 s+1} & \sum_{k=0}^{\infty} S_{k} d_{s-k}-\sum_{s=0}^{\infty} z^{2 s+2+r} \sum_{k=0}^{s} S_{k} c_{s-k} \\
= & \sum_{s=0}^{\infty}(s+t+1) c_{s} z^{2 s+r}-\sum_{s=0}^{\infty}(s+1) d_{s+1} z^{2 s+1}
\end{aligned}
$$

which yields the recurrence relation

$$
\sum_{k=0}^{l} S_{k}\left[c_{l-t-1-k}-d_{l-k}\right]=-(l+1)\left[c_{l-t}-d_{l+1}\right], \quad l=0,1,2, \ldots .
$$

As special cases we get

$$
\begin{aligned}
& S_{0}=\frac{1}{2}-\frac{a_{1}}{a_{0}}+\frac{1}{a_{0}} \delta_{t, 0}, \\
& S_{1}=\frac{1}{6}+\frac{a_{1}^{2}}{a_{0}}-\frac{2 a_{2}}{a_{0}}+\left[\frac{2}{3 a_{0}}+\frac{1-2 a_{1}}{a_{0}^{2}}+\frac{2 b_{1}}{a_{0}}\right] \delta_{t, 0}+2 \delta_{t, 1} .
\end{aligned}
$$

The problem in [1, Section 6] is a special case of this, with $t=0, a_{0}=-k$, $a_{l}=b_{l}=0$ for $l>0$. In this case, we get from (3.26), (3.8) and $b_{0}=1$,

$$
\sum_{s=0}^{l} \frac{(-1)^{s}(2 l-2 s-k)}{(2 l-2 s) !} S_{s}=\frac{2 l+2-k}{2[(2 l+1) !]},
$$

which coincides with $[1,(36)]$ for $S_{\imath} \equiv T_{\imath}(k), s=0,1,2, \cdots$.

4. Bessel Functions of Order One-Half. The Bessel function $J_{l+1 / 2}(z), l>0$, is given by [3, p. 298],

$$
J_{l+1 / 2}(z)=\left(\frac{2}{\pi z}\right)^{1 / 2}\left[R_{l, 1 / 2}(z) \sin z-R_{l-1,3 / 2}(z) \cos z\right]
$$

where [3, p. 296],

$$
R_{l, \nu}(z)=\sum_{n=0}^{[l / 2]}(-1)^{n}\left(\begin{array}{c}
l-n \\
n
\end{array}\right) \frac{\Gamma(\nu+l-n)}{\Gamma(\nu+n)}\left(\frac{1}{2} z\right)^{-l+2 n} .
$$

Also, [3, p. 40],

$$
J_{l+1 / 2}(z)=\left(\frac{1}{2} z\right)^{l+1 / 2} \sum_{k=0}^{\infty} \frac{(-1)^{k}(z / 2)^{2 k}}{k ! \Gamma(l+k+3 / 2)}=z^{l+1 / 2} P(z), \quad \text { where } P(0) \neq 0 .
$$

By (4.1), (4.3) we get 


$$
(\pi / 2)^{1 / 2} z^{2 l+1} P(z)=\left[z^{l} R_{l, 1 / 2}(z)\right] \sin z-\left[z^{l-1} R_{l-1,3 / 2}(z)\right] z \cos z .
$$

Now, $z^{l} R_{l, 1 / 2}(z)=2^{l} g_{l,-1 / 2}\left(\frac{1}{4} z^{2}\right)$, and $z^{l-1} R_{l-1,3 / 2}(z)=2^{l-1} g_{l-1,1 / 2}\left(\frac{1}{4} z^{2}\right)$, where

$$
g_{l, \nu}(\zeta)=\sum_{k=0}^{[l / 2]}\left(\begin{array}{c}
l-k \\
k
\end{array}\right) \frac{\Gamma(\nu+l-k+1)}{\Gamma(\nu+k+1)} \zeta^{k}
$$

are the modified Lommel polynomials [3, p. 303].

Since

$$
2^{l} g_{l,-1 / 2}(0)=\frac{2^{l} \Gamma\left(l+\frac{1}{2}\right)}{\Gamma\left(\frac{1}{2}\right)} \text { and } 2^{l-1} g_{l-1,1 / 2}(0)=\frac{2^{l-1} \Gamma\left(l+\frac{1}{2}\right)}{\Gamma\left(\frac{3}{2}\right)}=2^{l} g_{l,-1 / 2}(0)
$$

we get that

$$
\begin{aligned}
f_{l}(z) & =\frac{\Gamma\left(\frac{1}{2}\right)}{2^{l} \Gamma\left(l+\frac{1}{2}\right)}\left(\frac{\pi}{2}\right)^{1 / 2} z^{2 l+1} P(z) \\
& =\frac{\Gamma\left(\frac{1}{2}\right)}{2^{l} \Gamma\left(l+\frac{1}{2}\right)}\left(\frac{\pi}{2}\right)^{1 / 2} z^{l+1 / 2} J_{l+1 / 2}(z) \\
& =B_{m}\left(z^{2}\right) \sin z-z A_{n}\left(z^{2}\right) \cos z
\end{aligned}
$$

is of the form (3.4), with $r=1, b_{0}=a_{0}=1$,

$$
B_{m}\left(z^{2}\right)=\frac{\Gamma\left(\frac{1}{2}\right)}{\Gamma\left(l+\frac{1}{2}\right)} g_{l,-1 / 2}\left(\frac{1}{4} z^{2}\right)
$$

and

$$
A_{n}\left(z^{2}\right)=\frac{\Gamma\left(\frac{1}{2}\right)}{\Gamma\left(l+\frac{1}{2}\right)} g_{l-1,1 / 2}\left(\frac{1}{4} z^{2}\right)
$$

where

$$
n=m=(l-1) / 2, \quad l \text { odd, } \quad n=l / 2, \quad n=m-1, \quad l \text { even. }
$$

We can therefore apply the results of Section 3 to $J_{l+1 / 2}(z), l>0$. It is well known $\left[3\right.$, p. 482] that the Bessel function $J_{\nu}(z)$ has only real zeros for $\nu>-1$, and this is therefore true for $J_{l+1 / 2}(z), l>0$. The following theorem is therefore of interest.

THEOREM 3. If in $J_{l+1 / 2}(z)$, for $l>2$, we perturb any one of the coefficients in $R_{l, 1 / 2}(z)$ or $R_{l-1,3 / 2}(z)$, the resultant function has nonreal zeros.

To prove this theorem, we shall first prove two lemmas which are of interest themselves.

LEMMA 3. The coefficients $b_{0}, b_{1}, \cdots, b_{m}, a_{0}, a_{1}, \cdots, a_{n}$, of (4.7), (4.8) are uniquely determined by the following set of linear nonhomogenous equations

$$
b_{0}=1, \quad c_{i}=d_{i}, \quad j=0,1, \cdots, l-1,
$$

where $c_{i}, d_{i}$ are defined as in (3.8).

Proof. $b_{0}=1$ is a condition of (3.4), which is satisfied by $f_{l}(z)$ in (4.6). From (3.10) we have

$$
f_{l}(z)=C_{0} z^{Q} \prod_{k=1}^{\infty}\left(1-z^{2} / \alpha_{k}^{2}\right)=z^{Q} P_{1}(z)
$$


where $P_{1}(0) \neq 0$, and $q=2 i+1$ if $c_{i}-d_{i}=0$ for $j=0,1, \cdots, i-1$, and $c_{i}-d_{i} \neq 0$. From (4.6), $f_{l}(z)=z^{2 l+1} P_{2}(z)$, with $P_{2}(0) \neq 0$. It follows that $q=2 l+1$, and therefore $i=l$, and (4.10) is satisfied. The number of equations in (4.10) is $l+1$, and the number of unknowns (counting $b_{0}$ ) is $n+m+2$ which by (4.9) is equal to $l+1$, so that (4.10) is a set of $l+1$ nonhomogenous linear equations in $l+1$ unknowns. For uniqueness, see Appendix.

LEMMA 4. Let $\bar{b}_{0}(=1), \bar{b}_{1}, \cdots, \bar{b}_{m}, \bar{a}_{0}, \bar{a}_{1}, \cdots, \bar{a}_{n}$, be the (unique) solution of (4.10). Let $b_{\mathrm{s}}=\bar{b}_{\mathrm{i}}+\epsilon, a_{\mathrm{s}}=\bar{a}_{\mathrm{i}}+\delta, 0 \leqq s \leqq m$, and $b_{i}=\bar{b}_{i}, a_{i}=\bar{a}_{i}$ for $j \neq s$. [If $n=$ $m-1$, see (4.9), then $\bar{a}_{m}=\delta=0$.] Let

$$
f_{l}(z, s, \epsilon, \delta)=\frac{1}{b_{0}}\left[\sum_{k=0}^{m} b_{k} z^{2 k} \sin z-z \sum_{k=0}^{n} a_{k} z^{2 k} \cos z\right],
$$

and let $S_{k}^{l}(s, \epsilon, \delta)$ denote the sums of the nonzero zeros $(1.1)$ of $f_{l}(z, s, \epsilon, \delta)$. Then

$$
S_{i}^{l}(s, \epsilon, 0)=\pi^{-2 i-2} \zeta(2 j+2), \quad j \leqq l-s-2,
$$

and

$$
S_{i}^{l}(s, 0, \delta)=\pi^{-2 i-2}\left(2^{2 j+2}-1\right) \zeta(2 j+2), \quad j \leqq l-s-2 .
$$

In particular, when $j=0$, (4.13) holds for $l>2$, and $l=2, s=0$, and (4.14) holds for $l \geqq 2$.

Proof. $f_{l}(z, s, \epsilon, \delta)$ is of the form (3.4), with $r=1$. It follows from (3.8) and (4.10), that $c_{i}-d_{i}=0$ for $j<s$, and

$$
\begin{aligned}
c_{s}-d_{s} & =(-1)^{s}\left[\sum_{k=0}^{\dot{S}} \frac{(-1)^{k} \bar{b}_{k}}{(2 s-2 k+1) !}+(-1)^{s} \epsilon-\sum_{k=0}^{s} \frac{(-1)^{k} \bar{a}_{k}}{(2 s-2 k) !}-(-1)^{s} \delta\right] \\
& =\epsilon-\delta \quad(=\epsilon, \text { if } s=m=n+1) .
\end{aligned}
$$

For $\epsilon \neq \delta$, we can write, by (3.10),

$$
f_{l}(z, s, \epsilon, \delta)=(\epsilon-\delta) z^{2 s+1} \prod_{k=1}^{\infty}\left(1-z^{2} / \alpha_{k}^{2}\right),
$$

and by (3.22) we have

$$
\begin{array}{r}
\sum_{k=0}^{i} S_{k}^{l}(s, \epsilon, \delta)\left[c_{s+i-k}-d_{s+j-k}\right]=-(j+1)\left[c_{s+j+1}-d_{s+i+1}\right], \\
j=0,1,2, \cdots .
\end{array}
$$

If $s+t \leqq l-1$, then by (4.10) and (3.8),

$$
c_{s+t}-d_{s+t}=\frac{(-1)^{t}}{(2 t+1) !} \cdot \epsilon-\frac{(-1)^{t}}{(2 t) !} \cdot \delta,
$$

and so for $s+j+1 \leqq l-1$, (4.17) becomes

$$
\sum_{k=0}^{i} S_{k}^{l}(s, \epsilon, \delta)(-1)^{k}\left[\frac{\epsilon-(2 j-2 k+1) \delta}{(2 j-2 k+1) !}\right]=(j+1) \frac{\epsilon-(2 j+3) \delta}{(2 j+3) !},
$$

which is equation [1, (21)] with the parameter $k=\delta / \epsilon \neq 1$. In particular, if $\epsilon \neq 0$, $\delta=0$, this is [1, (21)] with $k=0$, and so by [1, (24)], we have (4.13). If $\delta \neq 0, \epsilon=0$, this is $[1,(21)]$ with $k \rightarrow \infty$, and so by $[1,(28)]$ we have (4.14). Since $s \leqq m$, it follows 
from (4.9) that when $j=0,(4.13)$ holds for $l>2$ and $l=2, s=0$, and since $s \leqq n$ in (4.14), (4.14) holds for $l \geqq 2$, if $j=0$.

Proof of Theorem 3. The nonzero zeros of $J_{l+1 / 2}(z)$ are the same as those of $f_{l}(z)$ in (4.6), and perturbing a coefficient of $R_{l, 1 / 2}(z)$ or $R_{l-1,3 / 2}(z)$ is equivalent to perturbing a coefficient of $B_{m}\left(z^{2}\right)$ or $A_{n}\left(z^{2}\right)$ in (4.6). We can therefore look at the function $f_{l}(z)$. Let $b_{i}(j=1,2, \cdots, m), a_{i}(j=1,2, \cdots, n)$ be defined as in Lemma 4. Since $l>2$, we have by (4.13)

$$
S_{0}^{l}(s, \epsilon, 0)=\pi^{-2} \zeta(2)=\frac{1}{6}, \quad \epsilon \neq 0,
$$

and by (4.14),

$$
S_{0}^{l}(s, 0, \delta)=\pi^{-2} 3 \zeta(2)=\frac{1}{2}, \quad \delta \neq 0 .
$$

From (4.20) it follows that

$$
\lim _{\epsilon \rightarrow 0} S_{0}^{l}(s, \epsilon, 0)=\frac{1}{6} .
$$

The roots $\pm \alpha_{1}, \pm \alpha_{2}, \cdots$ depend continuously on $\epsilon$. If $\epsilon \neq 0(\delta=0)$, we have from (4.16) that the multiplicity at zero is of order $2 s+1$, but when $\epsilon=0(\delta=0)$, we have from (4.6) that the multiplicity at zero is $2 l+1$. Since the roots occur in pairs $\pm \alpha$, we must have

$$
\lim _{\epsilon \rightarrow 0} \alpha_{i}^{2}=0, \quad j=1,2, \cdots, l-s
$$

But

$$
\frac{1}{6}=S_{0}^{l}(s, \epsilon, 0)=\sum_{k=1}^{l-s} \alpha_{k}^{-2}+\sum_{k=l-s+1}^{\infty} \alpha_{k}^{-2},
$$

and

$$
\lim _{\epsilon \rightarrow 0} \sum_{k=l-s+1}^{\infty} \alpha_{k}^{-2}=\frac{1}{2(2 l+3)},
$$

see [3, p. 502] or [4]. We therefore get that $Q(\epsilon)=\sum_{k=1}^{l-s} \alpha_{k}^{-2}$ stays finite as $\epsilon \rightarrow 0$ : If all $\alpha_{k}^{2}$ in $Q(\epsilon)$ tended to zero through positive values, then $\lim _{\epsilon \rightarrow 0} Q(\epsilon)=\infty$. So that, when $\epsilon \neq 0(|\epsilon| \ll 1)$, we must have at least one root, $\alpha_{i}$, for which $\alpha_{i}^{2}$ is not positive, i.e., $\alpha_{i}$ is nonreal. The same argument applied to $S_{0}^{l}(s, 0, \delta)$ by $(4.21)$ again yields that if $\delta \neq 0(|\delta| \ll 1)$ we must have nonreal roots, completing the proof.

Remark. From the proof and Lemma 4, it is obvious that Theorem 3 holds also when $l=2, s=0$. (For $l=2$, we only have $a_{0}, b_{0}$ and $b_{1}$, and the theorem does not apply to $b_{1}$.)

There are three cases which Theorem 3 does not cover, if $l+\frac{1}{2}>0$; the cases $l=0, l=1$, and $l=2, s=1$ (only $b_{1}$ ). The case $l=0$ is not of the form we are discussing. Indeed $(\pi z / 2)^{1 / 2} J_{1 / 2}(z)=\sin z$, so we only have $b_{0}=1$, and obviously the roots do not depend on changing $b_{0}$ to any nonzero constant. The other two cases are indeed different from the cases covered by Theorem 3, and we have the following.

THEOREM 4. Under the same definitions as in Lemma 4,

(1) $f_{1}(z, 0,-\epsilon, 0)$ and $f_{1}(z, 0,0, \delta)$ have only real zeros for $\epsilon \geqq 0, \delta \geqq 0$, and $a$ pair of imaginary roots for $\epsilon<0, \delta<0(|\epsilon| \ll 1,|\delta| \ll 1)$. 
(2) $f_{2}(z, 1,-\epsilon, 0)$ has only real roots for $\epsilon \geqq 0$, and a pair of imaginary roots for $\epsilon<0(|\epsilon| \ll 1)$.

Proof. Part (1). This is the problem of [1, Section 1], with the parameter $k=$ $a_{0} / b_{0}$ there, and (1) follows.

Part (2). Note that

$$
f_{2}(z)=\left(1-\frac{1}{3} z^{2}\right) \sin z-z \cos z .
$$

If we look at the function $g(z)=\left(k_{1}-k_{2} z^{2}\right) \sin z-z \cos z$, the roots, $\pm \alpha$ of $g(z)$ arise from the Sturm-Liouville system

$$
\begin{aligned}
u^{\prime \prime}+\alpha^{2} u & =0, \\
C u(0)+D u^{\prime}(0) & =0, \\
E u(1)-F u^{\prime}(1) & =0, \quad E D+C F \neq 0,
\end{aligned}
$$

where $k_{1}=C E /(E D+C F), k_{2}=F D /(E D+C F)$.

By Sturm-Liouville theory, $\alpha^{2}$ are all real, and therefore $f_{2}(z, 1,-\epsilon, 0)$ may have either real or imaginary roots. An imaginary root $z= \pm i x, x>0$, of $f_{2}(z, 1,-\epsilon, 0)$ should satisfy the equation

$$
y_{1}(x)=y_{2}(x),
$$

where $y_{1}(x)=\tanh x, y_{2}(x)=x /\left(1+c x^{2}\right), c=\frac{1}{3}+\epsilon$. Since $l-s=2-1=1$ only one such $x>0$ can exist (for $\epsilon$ small enough). $y_{1}(0)=y_{2}(0)=0$ and $\lim _{x \rightarrow \infty} y_{1}(x)=1, \lim _{x \rightarrow \infty} y_{2}(x)=0$. As $y_{1}(x), y_{2}(x)$ may intersect only once, for $x>0$, it is necessary and sufficient that $y_{2}(x)>y_{1}(x)$ in some interval $\left(0, \eta^{2}\right)$, for the existence of a positive solution of (4.26). Checking derivatives at zero, one finds $y_{1}^{(j)}(0)=y_{2}^{(j)}(0)$ for $j=0,1,2$, and $y_{1}^{(3)}(0)=-2, y_{2}^{(3)}(0)=-6 c$, and therefore, there exists a positive root of (4.26) iff $-6 c>-2$, i.e., $c<\frac{1}{3}$. This concludes the proof of Part (2).

To get an idea of the asymptotic behavior of the roots which split away from zero when $\epsilon$ (or $\delta$ ) tend to zero, note that by the definitions, $\lim _{\epsilon \rightarrow 0} f_{l}(z, s, \epsilon, 0)=$ $\lim _{\delta \rightarrow 0} f_{l}(z, s, 0, \delta)=f_{l}(z)$. From (4.16),

$$
\begin{aligned}
f_{l}(z, s, \epsilon, 0) & =\epsilon z^{2 s+1} \prod_{k=1}^{\infty}\left(1-z^{2} / \alpha_{k}^{2}\right) \\
& =z^{2 l+1} \prod_{k=l-s+1}^{\infty}\left(1-z^{2} / \alpha_{k}^{2}\right) \prod_{k=1}^{l-s}\left(1 / z^{2}-1 / \alpha_{k}^{2}\right) \epsilon \\
& =z^{2 l+1} \prod_{k=l-s+1}^{\infty}\left(1-z^{2} / \alpha_{k}^{2}\right) \prod_{k=1}^{l-s}\left(1-\alpha_{k}^{2} / z^{2}\right)(-1)^{l-s} \epsilon \prod_{k=1}^{l-s} \alpha_{k}^{-2} .
\end{aligned}
$$

Take the limit $\epsilon \rightarrow 0$ of (4.27) and use (4.23), (4.6), the continuous dependence of the roots on $\epsilon$, and (3.10), to get

$$
f_{l}(z)=f_{i}(z) \lim _{\epsilon \rightarrow 0}(-1)^{l-s} \epsilon\left(c_{l}-d_{l}\right)^{-1} \prod_{k=1}^{l-s} \alpha_{k}^{-2},
$$

or

$$
\lim _{\epsilon \rightarrow 0} \prod_{k=1}^{l-s} \alpha_{k}^{-2} \cdot \epsilon=(-1)^{l-s}\left(c_{l}-d_{l}\right)
$$


From (4.3), (4.6) we get that

$$
c_{l}-d_{l}=(2 l+1)^{-1}\left[2^{l} l ! /(2 l) !\right]^{2},
$$

and we get

$$
\lim _{\epsilon \rightarrow 0} \prod_{k=1}^{l-s} \alpha_{k}^{-2} \cdot \epsilon=(-1)^{l-s}(2 l+1)^{-1}\left[2^{l} l ! /(2 l) !\right]^{2} .
$$

Again, when $\epsilon=0, \delta \neq 0$, (4.29) holds with $\epsilon$ replaced by $(-\delta)$.

Example. $l=3, s=1, \epsilon<0$. By Theorem 3, we know we must have at least one nonreal root. By (4.29), $\lim _{\epsilon \rightarrow 0} \epsilon \alpha_{1}^{-2} \alpha_{2}^{-2}=1 / 1575$, and from Theorem 3, we have $\lim _{\epsilon \rightarrow 0} \alpha_{1}^{-2}+\alpha_{2}^{-2}=\frac{1}{9}$. If $|\epsilon| \ll 1$, we must therefore have $\alpha^{2}=\alpha_{1}^{2}>0$, and $-\beta^{2}=\alpha_{2}^{2}<0$, and so the two double roots at zero separate to $\pm \alpha(\alpha>0)$ along the real axis, and $\pm i \beta(\beta>0)$ along the imaginary axis. For $|\epsilon| \ll 1, \alpha^{2} \beta^{2} \approx 1575(-\epsilon)$, $\beta^{2}-\alpha^{2} \approx 175(-\epsilon)$, or $\alpha^{2} \approx \beta^{2} \approx 40|\epsilon|^{1 / 2}+O(\epsilon)$.

We have [3, p. 298],

$$
(-1)^{l} J_{-l-1 / 2}(z)=(2 / \pi z)^{1 / 2}\left[R_{l-1,3 / 2}(z) \sin z+R_{l, 1 / 2}(z) \cos z\right],
$$

and so

$$
\frac{\Gamma\left(\frac{1}{2}\right)}{2^{l} \Gamma\left(l+\frac{1}{2}\right)}(-1)^{l}\left(\frac{\pi}{2}\right)^{1 / 2} z^{l+1 / 2} J_{-l-1 / 2}(z), \quad l>0,
$$

is of the form (3.5) with $r=1, m=n=(l-1) / 2$ for $l$ odd and $n=l / 2, m=n-1$ for $l$ even, and the coefficients are given by

$$
b_{i}=\bar{a}_{i}, \quad a_{i}=-\bar{b}_{i}, \quad j=1,2, \cdots, n,
$$

where $\bar{a}_{i}, \bar{b}_{i}(j=1, \cdots, n)$ are the solutions of (4.10).

Since $r=1,(3.26)$ holds with $t=0$, and from (3.27), (4.31), we get

$$
S_{0}=-\frac{1}{2}-\bar{b}_{1}, \quad S_{1}=\frac{1}{2}+\bar{b}_{1}^{2}+2 \bar{b}_{2}+2 \bar{b}_{1}-2 \bar{a}_{1} \text {. }
$$

Appendix. We want to prove that there exists a unique solution $b_{0}, \cdots, b_{m}$, $a_{0}, \cdots, a_{n}$ to the set of equations

$$
\begin{gathered}
b_{0}=1, \\
c_{s}-d_{s}=(-1)^{s}\left[\sum_{k=0}^{m} \frac{(-1)^{k} b_{k}}{(2 s-2 k+1) !}-\sum_{k=0}^{n} \frac{(-1)^{k} a_{k}}{(2 s-2 k) !}\right]=0, \\
s=0,1,2, \cdots, l-1,
\end{gathered}
$$

$m=n=(l-1) / 2$, for $l$ odd, and $m=l / 2, n=m-1$ for $l$ even.

Note that we can replace $n$ by $m$, and add the equation $a_{m}=0$, for $l$ even. We know that for any $l \geqq 0$ there exists a solution to (A.1) given by the coefficients in (4.7), (4.8). From (3.9), (A.1) holds iff

$$
f(z)=\sin z \sum_{k=0}^{m} b_{k} z^{2 k}-z \cos z \sum_{k=0}^{n} a_{k} z^{2 k}, \quad\left(b_{0}=1\right),
$$

has a zero of multiplicity at least $2 l+1$ at zero, and actually, for the solution we 
know it is exactly of multiplicity $2 l+1$, by (4.28). Thus, (A.1) admits a unique solution iff any other choice of the coefficients, $b_{0}(=1), b_{1}, \cdots, b_{m}, a_{0}, a_{1}, \cdots, a_{n}$, would have the corresponding function $f(z)$ in (A.2), with a zero of multiplicity less than $2 l+1$, at zero. We therefore rephrase what we want to prove as follows:

THEOREM 5. There exists a unique set of coefficients $b_{0}, \cdots, b_{m}, a_{0}, \cdots, a_{m}$ such that, at zero, $f(z)$, defined by (A.2), has a zero of multiplicity $4 m+1(l=2 m)$ if we demand $a_{m}=0$, and multiplicity $4 m+3(l=2 m+1)$ otherwise, and such that $b_{0}=1$.

Proof. By induction.

(1) $m=0$. From (A.1), $b_{0}=1$ for $l=0$, and $a_{0}=b_{0}=1$ for $l=1$, and the solution is unique, so the theorem holds.

(2) The induction hypothesis is that the theorem is true for $m=0,1, \cdots, i$. We want to prove it for $m=i+1$. Note that the first two equations in (A.1) are always $b_{0}=1, a_{0}=b_{0}$, and so

$$
b_{0}=a_{0}=1 \text { for all } m>0 .
$$

Part a. Let $b_{0}, \cdots, \bar{b}_{i+1}, \bar{a}_{0}, \cdots, \bar{a}_{i}, \bar{a}_{i+1}[=0]$, and $b_{0}, \cdots, b_{i+1}, a_{0}, \cdots$, $a_{i+1}[=0]$ be two distinct solutions of (A.1) for $l=2 i+2$. It follows from (A.2) and (3.9) that we can write

$$
f_{1}(z)=\sin z \sum_{k=0}^{i+1} \bar{b}_{k} z^{2 k}-z \cos z \sum_{k=0}^{i} \bar{a}_{k} z^{2 k}=z^{4 i+5} R_{1}\left(z^{2}\right)
$$

and

$$
f_{2}(z)=\sin z \sum_{k=0}^{i+1} b_{k} z^{2 k}-z \cos z \sum_{k=0}^{i} a_{k} z^{2 k}=z^{4 i+5} R_{2}\left(z^{2}\right) .
$$

Subtract (A.5) from (A.4) to get

$$
\sin z \sum_{k=0}^{i+1}\left(\bar{b}_{k}-b_{k}\right) z^{2 k}-z \cos z \sum_{k=0}^{i}\left(\bar{a}_{k}-a_{k}\right) z^{2 k}=z^{4 i+5}\left[R_{1}\left(z^{2}\right)-R_{2}\left(z^{2}\right)\right],
$$

and, by (A.3), we must have

$$
a_{0}=\bar{a}_{0}=b_{0}=\bar{b}_{0}=1 .
$$

From (A.1) it follows that $\bar{b}_{k}-b_{k}=0$ for all $k \leqq j-1 \leqq m+1$ iff $\bar{a}_{k}-a_{k}=0$ for all $k \leqq j-1 \leqq m+1$. Suppose $\bar{b}_{k}-b_{k}=0$ for $k=0,1,2, \cdots, j-1$, and $b_{i}-b_{i} \neq 0 . j \geqq 1$ because of (A.7), and $j \leqq i$ since the two solutions are distinct, and $\vec{a}_{i+1}=a_{i+1}=0$. Divide (A.6) by $z^{2 i}$ to get

$$
\begin{gathered}
\sin z \sum_{k=0}^{i+1-i}\left[\bar{b}_{k+j}-b_{k+j}\right] z^{2 k}-z \cos z \sum_{k=0}^{i-i}\left[\bar{a}_{k+i}-a_{k+j}\right] z^{2 k} \\
=z^{4 i+5-2 i}\left[R_{1}\left(z^{2}\right)-R_{2}\left(z^{2}\right)\right] .
\end{gathered}
$$

Divide through by $b_{i}-b_{i}(\neq 0)$, and the left-hand side of (A.8) is of the form (A.2) with $0 \leqq m \leqq i+1-j \leqq i$, and $a_{m}=0$. From the induction hypothesis, it follows that the left-hand side of (A.8) can have a zero of multiplicity at most $4 m+1 \leqq$ $4 i+5-4 j$ at zero. But the right-hand side of (A.8) has multiplicity of at least $4 i+5-2 j>4 i+5-4 j$, a contradiction. Thus, (A.1) admits a unique solution for $l=2 i+2$. 
Part b. Let $\bar{b}_{0}, \cdots, \bar{b}_{i+1}, \bar{a}_{0}, \cdots, \bar{a}_{i+1}$ and $b_{0}, \cdots, b_{i+1}, a_{0}, \cdots, a_{i+1}$ be two distinct solutions of (A.1) for $l=2 i+3$. Repeat the same process as in Part a to get the equation

$$
\begin{gathered}
\sin z \sum_{k=0}^{i+1-i}\left[\bar{b}_{k+i}-b_{k+i}\right] z^{2 k}-\sum_{k=0}^{i+1-i}\left[\bar{a}_{k+i}-a_{k+j}\right] z^{2 k} \\
=z^{4 i+7-2 i}\left[R_{3}\left(z^{2}\right)-R_{4}\left(z^{2}\right)\right],
\end{gathered}
$$

and $1 \leqq j \leqq m+1$. Again divide by $\bar{b}_{i}-b_{i}(\neq 0)$, and get the left-hand side of (A.9) in the form (A.2) with $0 \leqq m \leqq i+1-j \leqq i$. Since we do not require $a_{m}=0$, we have by the induction hypothesis a zero of multiplicity at most $4 m+3 \leqq$ $4 i+7-4 j$ for the left-hand side of (A.9) at zero. The right-hand side has, at zero, a zero of multiplicity at least $4 i+7-2 j>4 i+7-4 j$, since $j \geqq 1$, a contradiction. This concludes the proof.

Department of Mathematics and Statistics

Colorado State University

Fort Collins, Colorado 80521

Current address:

Department of Applied Mathematics

Weizmann Institute of Science

Rehovot, Israel

1. N. LIRON, "Some infinite sums," SIAM J. Math. Anal., v. 2, 1971, pp. 105-112.

2. R. P. BoAs, Entire Functions, Academic Press, New York, 1954. MR 16, 914.

3. G. N. WaTSON, $A$ Treatise on the Theory of Bessel Functions, 2nd ed., Cambridge Univ. Press, Cambridge; Macmillan, New York, 1944. MR 6, 64.

4. N. KishORE, "The Rayleigh function," Proc. Amer. Math. Soc., v. 14, 1963, pp. 527533. MR 27 \#1633. 